Supporting Information:

\title{
Monolayer Iridium Sulfide Halides with High Mobility \\ Transport Anisotropy and Highly Efficient Light Harvesting
}

Yang Song, ${ }^{a, b}$ Jinbo Pan, ${ }^{* a, b}$ Yan-Fang Zhang, ${ }^{a}$ Haitao Yang ${ }^{a}$ and Shixuan Du ${ }^{* a, b, c, d}$

a Institute of Physics \& University of Chinese Academy of Sciences, Chinese Academy of Sciences, Beijing 100190, China.

${ }^{\mathrm{b}}$ Beijing National Laboratory for Condensed Matter Physics, Beijing 100190, China.

${ }^{\mathrm{c}}$ CAS Center for Excellence in Topological Quantum Computation, Beijing 100190, China

d Songshan Lake Materials Laboratory, Dongguan 523808, China

\section{Content:}

\section{Calculation Methods}

2. Table S1: Lattice constants (a, b), thicknesses (d) of ML IrSX' at PBE level

3. Figure S1: Phonon spectra of ML IrSX' at PBE level.

4. Figure S2: Snapshot taken from the end of the AIMD simulation of ML IrSX's at $300 \mathrm{~K}$ for 5 ps at PBE level.

5. Figure S3: Band structure of ML IrSX' calculated with PBE functional.

6. Figure S4: Ferroelastic switching in ML IrSF.

\section{References}




\section{Calculation Methods}

DFT calculations. Density-functional-theory calculations are performed using projector-augmented wave $(\mathrm{PAW})^{1}$ pseudopotentials in conjunction with the PerdewBurke-Ernzerhof $(\mathrm{PBE})^{2}$ exchange-correlation functional as implemented in the Vienna Ab-initio Simulation Package (VASP). ${ }^{3-4}$ The energy cutoff of plane-wave basis is set to $600 \mathrm{eV}$. The thicknesses of vacuum length are larger than $15 \AA$. All the structural are relaxed until the force on each atom is less than $0.001 \mathrm{eV} \AA^{-1}$ and the break condition of the electronic self-consistent loop was set at $1 \times 10^{-7} \mathrm{eV}$. The $\mathrm{k}$ points sampling is $\Gamma$-centered $14 \times 10 \times 1$. The solid-state nudged elastic band (ssNEB) method $^{5}$ was used to search the ferroelastic transition pathway. To check the reliability of the electronic properties, the hybrid functional (HSE06) $)^{6-7}$ are used to optimize the structure, calculate the band structures and the carrier mobility for IrSF. For the HSE06 calculations, the energy cutoff of plane-wave basis is set to $400 \mathrm{eV}$. All the structural models are relaxed until the force on each atom is less than $0.001 \mathrm{eV} \AA^{-1}$ and the break condition of the electronic self-consistent loop was set at $1 \times 10^{-6} \mathrm{eV}$. The k-points sampling is $\Gamma$-centered $8 \times 6 \times 1$.

Stability calculations. To verify the dynamical stability of $\mathrm{MXX}^{\prime}$ monolayers, phonon dispersion analysis was performed by using the density functional perturbation theory method ${ }^{8-9}$ as implemented in the Phonopy code,${ }^{10}$ interfaced with VASP. The convergence criterion for energy is $10^{-8} \mathrm{eV}$. For the ab initio molecular dynamics (MD) simulations, a canonical (NVT) ensemble was used ${ }^{11}$. Time step for MD simulations is 1 fs. 
The thermodynamic stabilities of MXX' monolayers are evaluated by computing the $0 \mathrm{~K}, 0 \mathrm{~atm}$ ternary phase diagram. The compounds except for the predicted MXX' compounds in the phase diagram and their formation enthalpies are all obtained from the Materials Project database ${ }^{12}$. Total energies of all known compounds in the Materials Project are retrieved using the Materials Project REST API, ${ }^{13}$ Materials Project data were retrieved in January 2021. The formation enthalpies of MXX' monolayers are calculated by

$$
\Delta H=\frac{E\left(A_{x} B_{y} C_{z}\right)-x E(A)-y E(B)-z E(C)}{x+y+z}+\Delta c
$$

where $E(A), E(B)$, and $E(C)$ are the chemical potentials of $\mathrm{A}, \mathrm{B}$, and $\mathrm{C}$ elements under their ground states, respectively. The scheme of energy correction $\Delta c$, as described in previous literatures, ${ }^{14-15}$ is used to correct the overbinding of gas molecules and to adjust the formation enthalpies of mixing GGA and GGA+U calculations.

For the formation enthalpies calculations of $\operatorname{IrSX}$ bulk materials, interlayer interaction corrections are further considered, the formation enthalpies can thus be rewritten as

$$
\Delta H=\frac{E\left(A_{x} B_{y} C_{z}\right)-x E(A)-y E(B)-z E(C)}{x+y+z}+\Delta c+\Delta \text { inter }
$$

where $E\left(\operatorname{Ir} S X^{\prime}\right)$ is still the total energy of $\operatorname{IrSX}$ monolayer obtained using the PBE functional. To calculate the interlayer interaction sinter, a non-local correction function DFT-D3 ${ }^{16}$ is employed to consider the van der Waals interaction, $\Delta$ inter $=$ $E($ bulk $)-E($ monolayer $)$, where $E($ bulk $)$, and $E($ monolayer $)$ are van der Waals corrected total energies for bulk, and monolayer materials, respectively. Ternary phase 
diagram is computed using the pymatgen.analysis.phase_diagram module of the convex hull phase diagram analysis package in Pymatgen. ${ }^{17}$

Carrier mobility calculations. With the deformation potential theory and effective mass approximation, the carrier mobility of $2 \mathrm{D}$ system is expressed as $\mu_{2 D}=$ $\frac{e \hbar^{3} C_{2 D}}{k_{B} T m_{e}^{*} m_{d} E_{d}^{2}}$. The term $m_{e}^{*}$ is effective mass, which is measured from the band structure using the formula $m_{e(h)}^{*}= \pm \hbar^{2}\left(\frac{d^{2} E_{k}}{d k^{2}}\right)^{-1}$. The value of $\left(\frac{d^{2} E_{k}}{d k^{2}}\right)$ is obtained by parabolic fitting of energy $E_{k}$ versus to reciprocal vector $k$. The average effective mass $m_{d}$ is defined as $m_{d}=\sqrt{m_{x}^{*} m_{y}^{*}}$. The elastic modulus $C_{2 D}$ is derived from $C_{2 D}\left(\Delta l / l_{0}\right)^{2} / 2=(E$ $\left.-E_{0}\right) / S_{0 .}{ }^{18}$ Here, $C_{2 D}=\frac{1}{s_{0}} \frac{\partial^{2}\left(E-E_{0}\right)}{\partial \varepsilon^{2}}$ can be calculated by fitting a quadratic function relation of the change of the total energy $\left(E-E_{0}\right)$ and the uniaxial strains $\left(\varepsilon=\Delta l / l_{0}, \varepsilon\right.$ $\sim \pm 2 \%, \pm 1 \%, 0 \%) . S_{0}$ is the lattice area in the $x y$ plane, which is employed as the lattice volume at equilibrium for a $2 \mathrm{D}$ system. The deformation potential constant $E_{d}=$ $\mathrm{d} E_{\text {edge }} / d \varepsilon$ is calculated by fitting a linear relation of the band edges $\left(E_{\text {edge }}\right)$ and applied uniaxial strains $(\varepsilon)$. $T$ is set to room temperature, $300 \mathrm{~K}$. The above formula only considers the electron-phonon coupling and neglects external factors in actual situation. This simplified method results in overestimated carrier mobility in our calculations. ${ }^{19}$ However, the order of magnitude estimation of the mobility, and relative comparisons of mobilities in different materials are probably reliable. ${ }^{20}$

The electron-phonon scattering limited carrier mobilities of monolayer IrSF was calculated by using the PERTURBO software. ${ }^{21}$ The software can simulate the ultrafast nonequilibrium electron dynamics in the presence of electron-phonon scattering by using results from density functional theory and density functional perturbation theory 
calculations as input, and employing Wannier interpolation to reduce the computational cost. We construct 34 Wannier functions using sp ${ }^{3}$ orbitals on $\mathrm{S}$ and $\mathrm{F}$ atoms, and spd orbitals on Ir atom, and obtain the e-ph matrix elements in the Wannier basis using coarse $30 \times 30 \times 1 \mathbf{k}$-points grid and $6 \times 6 \times 1$ q-points grid. For the electron-phonon scattering limited mobilities calculations, the Brillouin zone grid of $150 \times 150 \times 1$ is employed for both $\mathbf{k}$ - and q-points. The relaxation time approximation (RTA), normconserving pseudopotentials are used, and the SOC effect is not considered.

Optical absorption spectra calculations. Absorption spectra are obtained by calculating the dielectric function. The expression of absorbance is $A(\omega)=1-$ $e^{-\alpha(\omega) \cdot \Delta z}$, in which $\quad \alpha(\omega)=\frac{\sqrt{2} \omega}{c}\left[\sqrt{\varepsilon_{1}^{2}+\varepsilon_{2}^{2}}-\varepsilon_{1}\right]^{\frac{1}{2}}$ is the absorption coefficient. The $\varepsilon_{1}$ and $\varepsilon_{2}$ are the real and imaginary parts of the dielectric function. $\omega$ is the light frequency. $c$ is the speed of light in vacuum. $\Delta \mathrm{z}$ is the unit-cell size in the $z$ direction. The electronic structures used in this optical absorption spectra calculation are obtained from HSE06 calculations. Excitonic effect was not considered. The total number of bands considered was set to be five times larger than that used in the calculations for electronic properties. 
Table S1. Lattice constants (a, b), thicknesses (d) of ML IrSX's at PBE level.

\begin{tabular}{cccc}
\hline $\operatorname{IrSX}^{\prime}$ & $\mathrm{a}(\AA)$ & $\mathrm{b}(\AA)$ & $\mathrm{d}(\AA)$ \\
\hline $\mathrm{IrSF}$ & 3.42 & 4.64 & 4.74 \\
$\mathrm{IrSCl}$ & 3.55 & 4.64 & 5.42 \\
$\mathrm{IrSBr}$ & 3.62 & 4.64 & 5.70 \\
$\mathrm{IrSI}$ & 3.74 & 4.64 & 5.98 \\
\hline
\end{tabular}


(a)

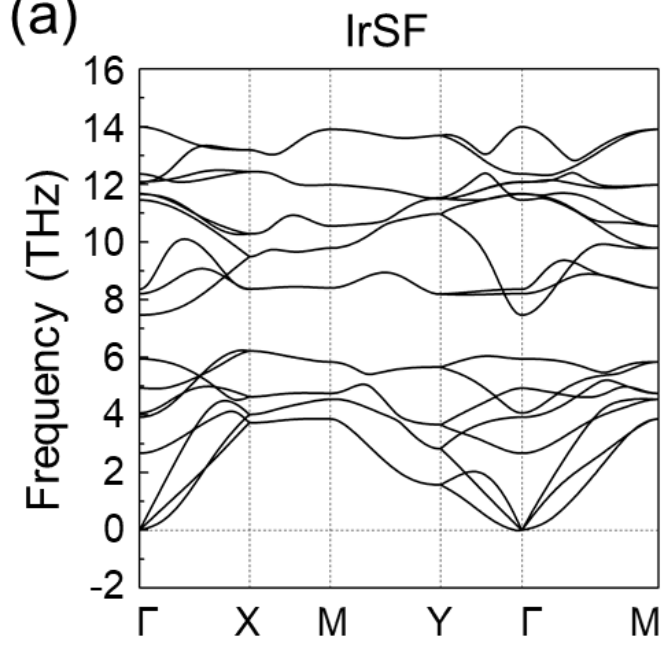

(c)

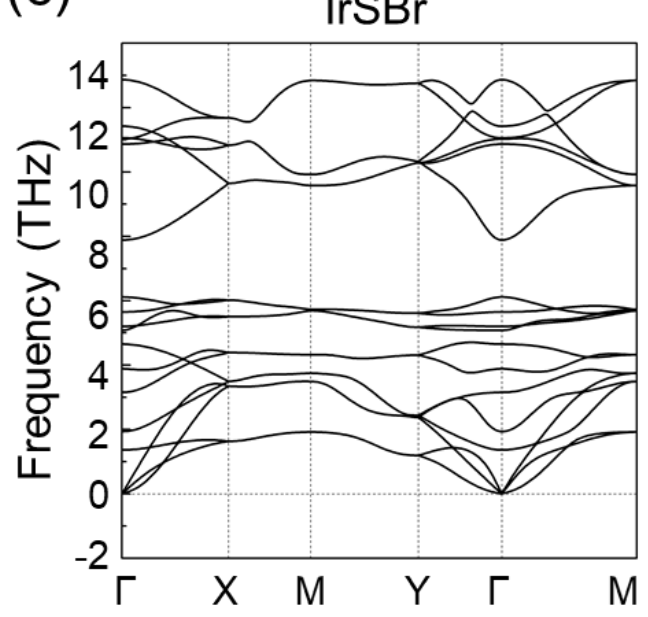

(b)

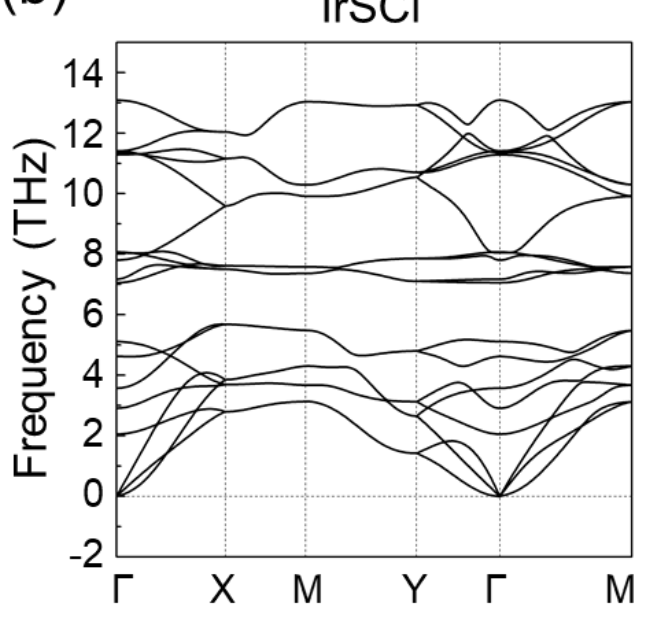

(d)

$|r S|$

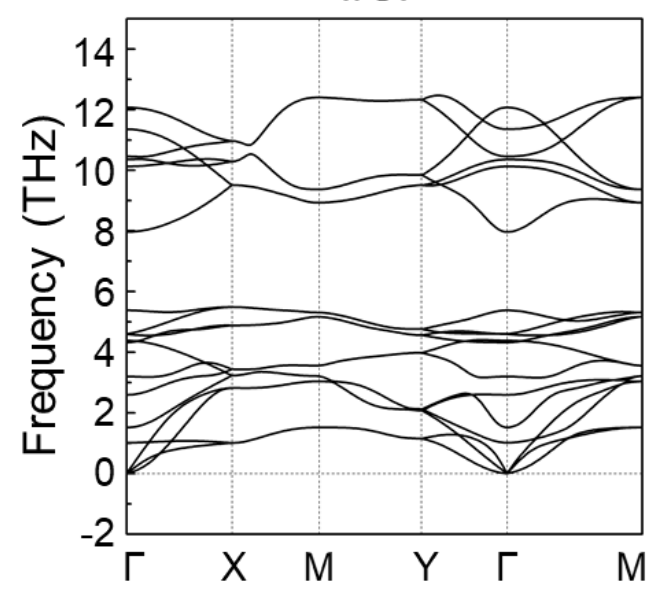

Figure S1. Phonon spectra of ML IrSX' at PBE level. No imaginary frequency is observed in phonon spectra, indicating ML IrSX's are dynamically stable. 

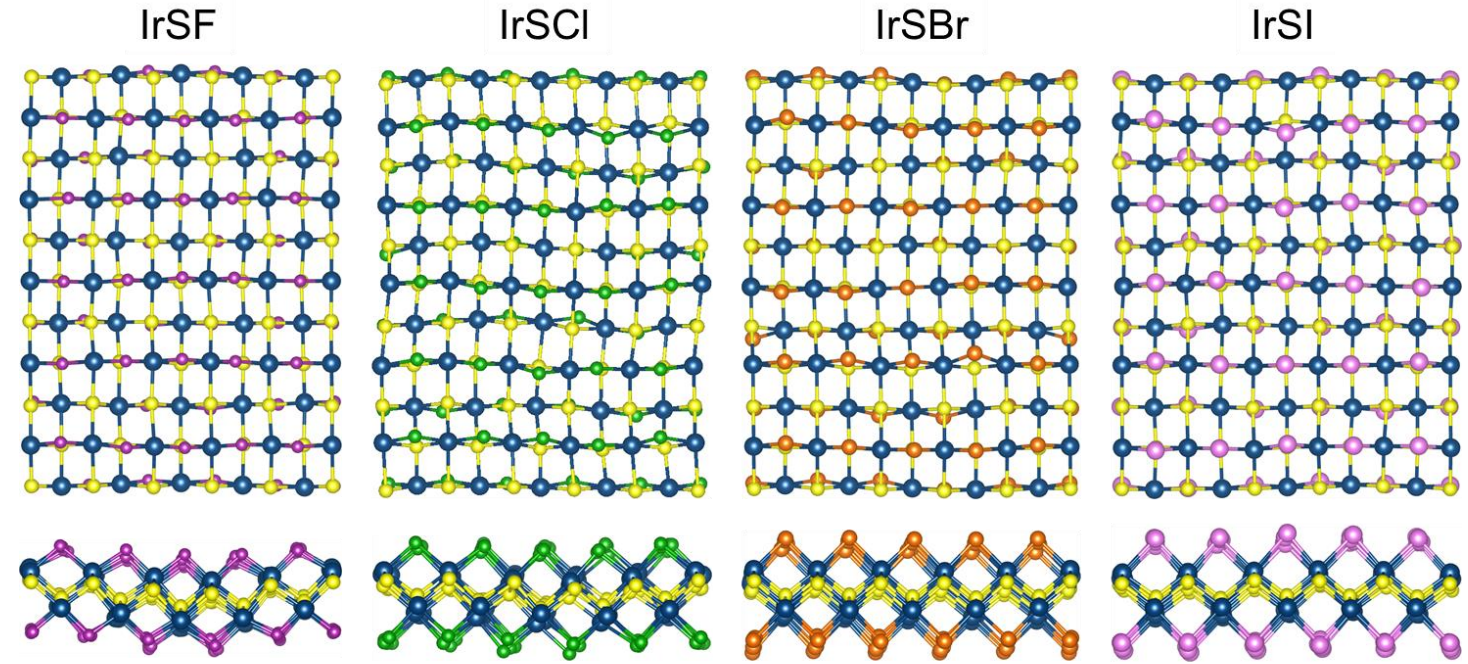

$\begin{array}{llllll}\bullet & 0 & \bullet & 0 & 0 & 0 \\ \mathrm{Ir} & \mathrm{S} & \mathrm{F} & \mathrm{Cl} & \mathrm{Br} & \mathrm{I}\end{array}$

Figure S2. Snapshots taken from the end of the AIMD simulations of ML IrSX's at 300

$\mathrm{K}$ for 5 ps at PBE level. 
(a)

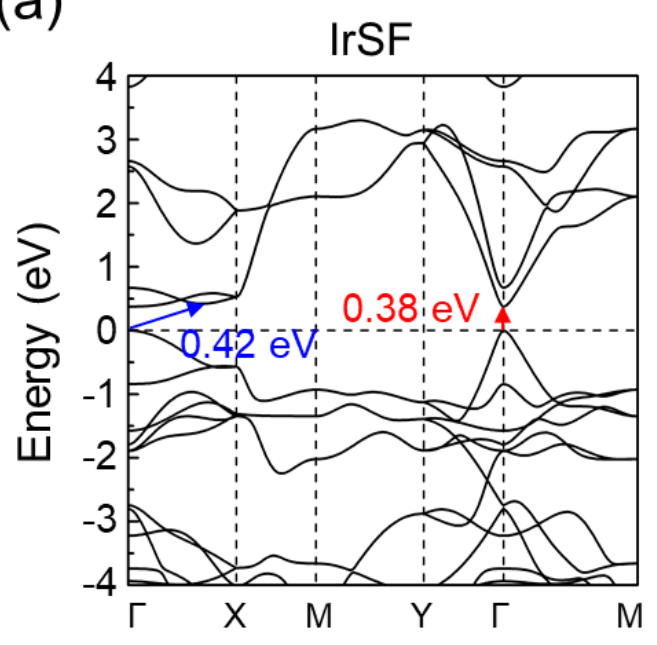

(c)

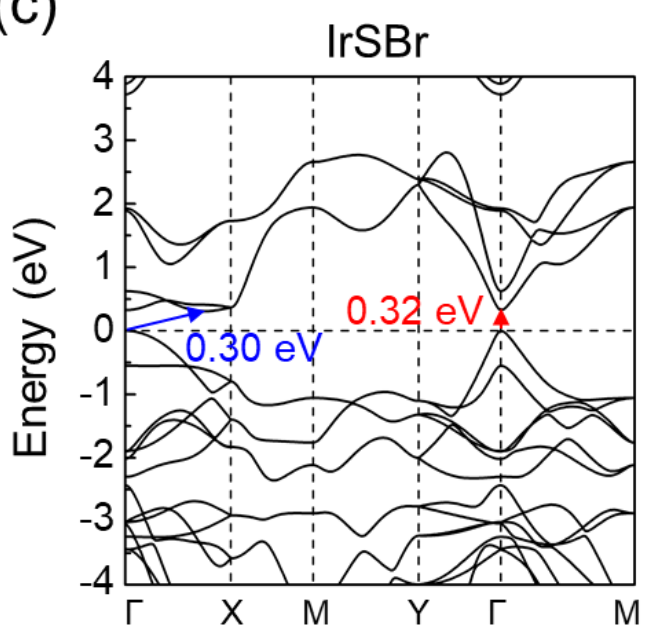

(b)

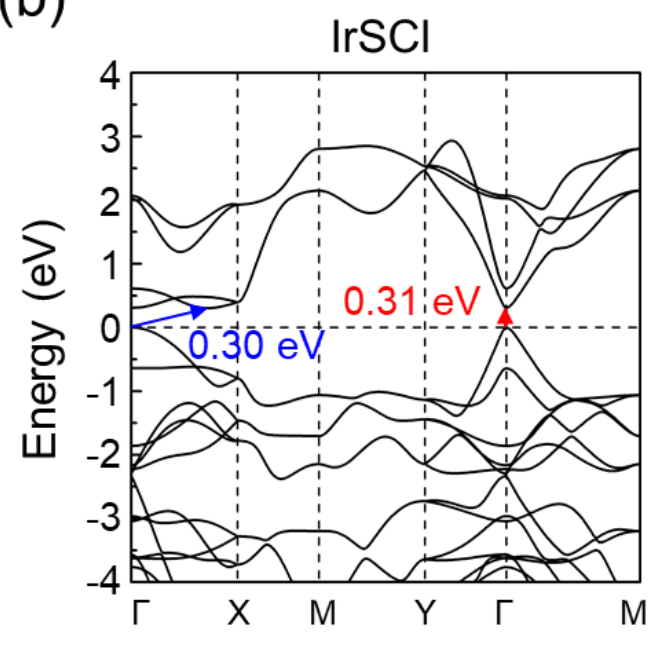

(d)

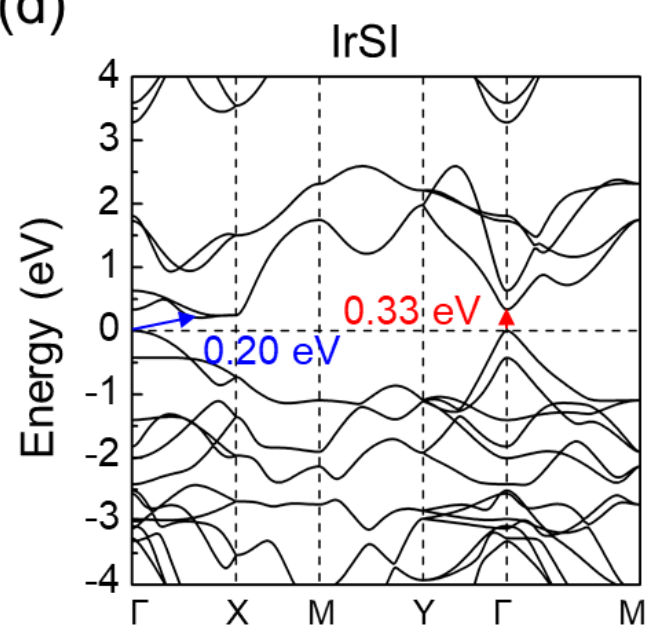

Figure S3. Band structures of ML IrSX's calculated with PBE functional. ML IrSF (a) is a direct band gap semiconductor, while ML $\operatorname{IrSCl}(\mathrm{b}), \operatorname{IrSBr}(\mathrm{c})$ and $\operatorname{IrSI}(\mathrm{d})$ are indirect band gap semiconductors, in which the direct band gaps are only slightly larger than the indirect ones. 

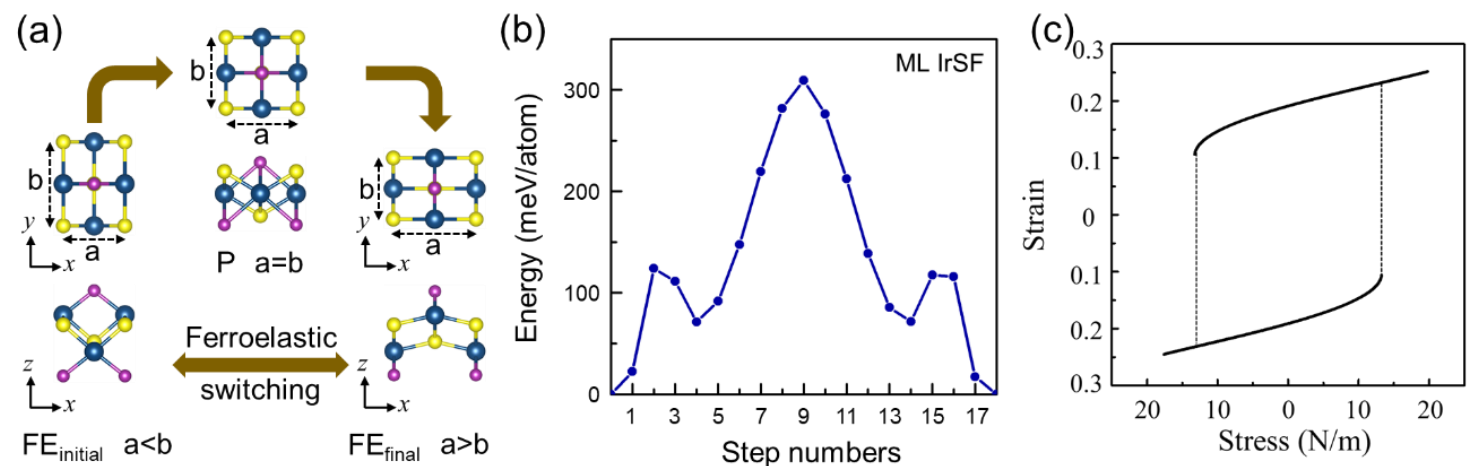

Figure S4. Ferroelastic switching in ML IrSF. (a) Pathway of ferroelastic transition for ML IrSF. (b) Energy barrier of ferroelastic switching as a function of step number in the nudged elastic band (NEB) for ML IrSF. (c) Force hysteresis loop is obtained by employing equilibrium principles. $^{22}$ The equilibrium state of the material is the strain value that yields a minimum Gibbs free energy given a stress at a fixed $0 \mathrm{~K}$.

The configurations of two ferroelastic ground states $\mathrm{FE}_{\text {initial }}$ and $\mathrm{FE}_{\text {final }}$ and the paraelastic state $\mathrm{P}$ are presented in Figure $\mathbf{S 4 a}$. The initial state $\mathrm{FE}_{\text {initial }}(\mathrm{a}<\mathrm{b})$ and the final state $\mathrm{FE}_{\text {final }}(\mathrm{a}>\mathrm{b})$ possess the same atomic structure with a $90^{\circ}$ rotation. Figure S4b shows the transition path of ferroelastic switching of ML IrSF. The path from the initial state $\left(\mathrm{FE}_{\text {initial }}\right)$ to the intermediate state $(\mathrm{P})$ is the same as the path from the intermediate state $(\mathrm{P})$ to the final state $\left(\mathrm{FE}_{\text {final }}\right)$ due to these symmetric structures with a $90^{\circ}$ rotation. The calculated ferroelastic switching barrier is $0.31 \mathrm{eV}$ per atom. 


\section{References}

(1) Blöchl, P. E. Projector Augmented-Wave Method. Phys. Rev. B 1994, 50, 17953 17979.

(2) Perdew, J. P.; Burke, K.; Ernzerhof, M. Generalized Gradient Approximation Made Simple. Phys. Rev. Lett. 1996, 77, 3865.

(3) Kresse, G.; Furthmüller, J. Efficiency of Ab-Initio Total Energy Calculations for Metals and Semiconductors Using a Plane-Wave Basis Set. Comput. Mater. Sci. 1996, 6, 15-50.

(4) Kresse, G.; Furthmüller, J. Efficient Iterative Schemes for Ab Initio Total-Energy Calculations Using a Plane-Wave Basis Set. Phys. Rev. B 1996, 54, 11169-11186.

(5) Sheppard, D.; Xiao, P.; Chemelewski, W.; Johnson, D. D.; Henkelman, G. A Generalized Solid-State Nudged Elastic Band Method. J. Chem. Phys. 2012, 136, 074103.

(6) Heyd, J.; Scuseria, G. E.; Ernzerhof, M. Hybrid Functionals Based on a Screened Coulomb Potential. J. Chem. Phys. 2003, 118, 8207-8215.

(7) Heyd, J.; Scuseria, G. E.; Ernzerhof, M. Erratum: "Hybrid Functionals Based on a Screened Coulomb Potential" [J. Chem. Phys. 118, 8207 (2003)]. J. Chem. Phys. 2006, $124,219906$.

(8) Baroni, S.; Giannozzi, P.; Testa, A. Green's-Function Approach to Linear Response in Solids. Phys. Rev. Lett. 1987, 58, 1861-1864.

(9) Giannozzi, P.; de Gironcoli, S.; Pavone, P.; Baroni, S. Ab Initio Calculation of Phonon Dispersions in Semiconductors. Phys. Rev. B 1991, 43, 7231-7242.

(10) Togo, A.; Oba, F.; Tanaka, I. First-Principles Calculations of the Ferroelastic Transition between Rutile-Type and $\mathrm{CaCl}_{2}$-Type $\mathrm{SiO}_{2}$ at High Pressures. Phys. Rev. $B$ 2008, 78,134106 .

(11) Nosé, S. A Unified Formulation of the Constant Temperature Molecular Dynamics Methods. J. Chem. Phys. 1984, 81, 511-519.

(12) Jain, A.; Ong, S. P.; Hautier, G.; Chen, W.; Richards, W. D.; Dacek, S.; Cholia, S.; Gunter, D.; Skinner, D.; Ceder, G.; et al. Commentary: The Materials Project: A 
Materials Genome Approach to Accelerating Materials Innovation. APL Mater. 2013, 1,011002 .

(13) Ong, S. P.; Cholia, S.; Jain, A.; Brafman, M.; Gunter, D.; Ceder, G.; Persson, K. A. The Materials Application Programming Interface (API): A Simple, Flexible and Efficient API for Materials Data Based on REpresentational State Transfer (REST) Principles. Comput. Mater. Sci. 2015, 97, 209-215.

(14) Wang, L.; Maxisch, T.; Ceder, G. Oxidation Energies of Transition Metal Oxides within the GGA+U Framework. Phys. Rev. B 2006, 73, 195107.

(15) Jain, A.; Hautier, G.; Ong, S. P.; Moore, C. J.; Fischer, C. C.; Persson, K. A.; Ceder, G. Formation Enthalpies by Mixing GGA and GGA + U Calculations. Phys. Rev. B 2011, 84,045115 .

(16) Grimme, S.; Antony, J.; Ehrlich, S.; Krieg, H. A Consistent and Accurate Ab Initio Parametrization of Density Functional Dispersion Correction (DFT-D) for the 94 Elements H-Pu. J. Chem. Phys. 2010, 132, 154104.

(17) Ong, S. P.; Richards, W. D.; Jain, A.; Hautier, G.; Kocher, M.; Cholia, S.; Gunter, D.; Chevrier, V. L.; Persson, K. A.; Ceder, G. Python Materials Genomics (Pymatgen): A Robust, Open-Source Python Library for Materials Analysis. Comput. Mater. Sci. 2013, 68, 314-319.

(18) Qiao, J.; Kong, X.; Hu, Z.-X.; Yang, F.; Ji, W. High-Mobility Transport Anisotropy and Linear Dichroism in Few-Layer Black Phosphorus. Nat. Commun. 2014, 5, 4475. (19) Fei, R.; Yang, L. Strain-Engineering the Anisotropic Electrical Conductance of Few-Layer Black Phosphorus. Nano Lett. 2014, 14, 2884-2889.

(20) Haastrup, S.; Strange, M.; Pandey, M.; Deilmann, T.; Schmidt, P. S.; Hinsche, N. F.; Gjerding, M. N.; Torelli, D.; Larsen, P. M.; Riis-Jensen, A. C.; et al. The Computational 2D Materials Database: High-Throughput Modeling and Discovery of Atomically Thin Crystals. 2D Mater. 2018, 5, 042002.

(21) Zhou, J.-J.; Park, J.; Lu, I. T.; Maliyov, I.; Tong, X.; Bernardi, M. Perturbo: A Software Package for Ab Initio Electron-Phonon Interactions, Charge Transport and Ultrafast Dynamics. Compt. Phys. Commun. 2021, 264, 107970. 
(22) Massad, J. E.; Smith, R. C. A Domain Wall Model for Hysteresis in Ferroelastic Materials. J. Intell. Mater. Syst. Struct. 2003, 14, 455-471. 